\title{
A LATTICE OF NORMAL SUBGROUPS THAT IS NOT EMBEDDABLE INTO THE SUBGROUP LATTICE OF AN ABELIAN GROUP
}

\author{
E. W. KISS and P. P. PÁLFY
}

\section{Introduction.}

In this paper we give a negative solution to the following problem of Bjarni Jónsson:

PROBLEM. Is the lattice of normal subgroups of every group embeddable into the subgroup lattice of an abelian group?

The problem goes back to the famous 1953 paper of Jonsson [J] (see the last sentence of the text there), and it is also mentioned in the third edition of Birkhoff's Lattice Theory [B] (Problem 63, p. 179).

We give a group of order $2^{9}$ whose lattice of normal subgroups does not have the desired embedding.

THEOREM. The lattice of normal subgroups of the three generator free group $G$ in the group variety defined by the laws $x^{4}=1$ and $x^{2} y=y x^{2}$ cannot be embedded into the subgroup lattice of any abelian group.

We obtained this negative solution in 1988 (see the account given by McKenzie [M], p. 42), but the publication of the result has been delayed. Meanwhile the second author and Csaba Szabó [PSz1], [PSz2] have obtained a stronger result by exhibiting a lattice identity valid in subgroup lattices of all abelian groups that fails in the lattice of normal subgroups of a certain group of order $2^{20}$. For the lattice of normal subgroups $\mathscr{N}(G)$ of our group $G$ this identity, however, does hold. We do not know, whether $\mathscr{N}(G)$ belongs to the lattice variety generated by the subgroup lattices of abelian groups or not. Our result shows only that it does not belong to the quasivariety generated by them.

\footnotetext{
* Research of both authors was partially supported by the Hungarian National Science Foundation, grant nos. 7442 and 16432.

Received July 24, 1996.
} 
Our notation is mostly standard. For basic results from group theory used here the reader may consult $[\mathrm{A}]$. The lattice of normal subgroups of a group $G$ will be denoted by $\mathscr{N}(G)$. If $G$ is abelian, then $\mathscr{N}(G)$ is simply the subgroup lattice of $G$. For normal subgroups $A \subseteq B \subseteq G$ we shall denote the interval in $\mathscr{N}(G)$ consisting of the normal subgroups $N$ with $A \subseteq N \subseteq B$ by $I[A, B]$. Sometimes we shall treat abelian groups of exponent $k$ as $Z_{k^{-}}$ modules. For a group $G$ and a natural number $m$ we use $G^{m}$ to denote the subgroup generated by all $m$-th powers in $G$. If $G$ is an additively written abelian group then we write $m G=\{m g \mid g \in G\}$ instead. (Notice that these elements already form a subgroup.)

\section{The group $G$.}

As it was mentioned in the Introduction we consider the (relatively) free group $G$ on three generators in the group variety defined by the laws

$$
x^{4}=1, \quad x^{2} y=y x^{2} .
$$

So the square of every element belongs to the center $\mathbf{Z}(G)$ of $G$. Notice that the commutators $[x, y]=x^{-1} y^{-1} x y=\left(x^{-1}\right)^{2}\left(x y^{-1}\right)^{2} y^{2}$ also belong to the center and have order at most two. From these observations it follows that $[x y, z]=[x, z][y, z],(x y)^{2}=x^{2} y^{2}[x, y]$, and $[y, x]=[x, y]$ (see [A], p. 26). Notice that our variety contains both the 8-element dihedral group and the quaternion group (in fact each of these groups generates the variety). Let the generators of $G$ be $a, b$, and $c$. It is easy to verify that every element of $G$ can be written in the form

$$
a^{\alpha} b^{\beta} c^{\gamma}[a, b]^{\rho}[a, c]^{\sigma}[b, c]^{\tau},
$$

where $0 \leq \alpha, \beta, \gamma<4,0 \leq \rho, \sigma, \tau<2$. By constructing appropriate homomorphisms into the quaternion group it is also straightforward to see that the above form of the elements of $G$ is unique. Thus, the order of $G$ is $2^{9}$. We have that the center $\mathbf{Z}(G)$ is elementary abelian of order $2^{6}$ with basis $a^{2}, b^{2}, c^{2},[a, b],[a, c],[b, c]$. The commutator subgroup $G^{\prime}$ has order $2^{3}$, the factor group $G / G^{\prime}$ is the direct product of three cyclic groups of order four. Furthermore, we have $2\left(G / G^{\prime}\right)=\mathbf{Z}(G) / G^{\prime}$.

\section{The lattice of normal subgroups.}

To shorten notation let $Z=\mathbf{Z}(G)$ denote the center. Now the factor group $G / Z$ is elementary abelian of order $2^{3}$, so it contains seven minimal subgroups $U_{i} / Z(i=1, \ldots, 7)$. Each $U_{i}$ is abelian. Let $V_{i}=U_{i}^{2}\left[U_{i}, G\right]$. 
Let us list all $U_{i}$ 's and the corresponding $V_{i}$ 's by giving their generators.

$$
\begin{aligned}
U_{1} & =\langle Z, a\rangle & V_{1} & =\left\langle a^{2},[a, b],[a, c]\right\rangle \\
U_{2} & =\langle Z, b\rangle & V_{2} & =\left\langle b^{2},[a, b],[b, c]\right\rangle \\
U_{3} & =\langle Z, c\rangle & V_{3} & =\left\langle c^{2},[a, c],[b, c]\right\rangle \\
U_{4} & =\langle Z, a b\rangle & V_{4} & =\left\langle a^{2} b^{2}[a, b],[a, b],[a, c][b, c]\right\rangle \\
U_{5} & =\langle Z, a c\rangle & V_{5} & =\left\langle a^{2} c^{2}[a, c],[a, c],[a, b][b, c]\right\rangle \\
U_{6} & =\langle Z, b c\rangle & V_{6} & =\left\langle b^{2} c^{2}[b, c],[a, b][a, c],[b, c]\right\rangle \\
U_{7} & =\langle Z, a b c\rangle & V_{7} & =\left\langle a^{2} b^{2} c^{2}[a, b][a, c][b, c],[a, b][a, c],[a, b][b, c]\right\rangle
\end{aligned}
$$

LEMMA 3.1. For each $1 \leq i \leq 7$ every subgroup between $V_{i}$ and $U_{i}$ is normal in G. Moreover, $U_{i} / V_{i}$ is elementary abelian of order $2^{4}$.

Proof. Let $V_{i} \subseteq H \subseteq U_{i}$. Since $[H, G] \subseteq\left[U_{i}, G\right] \subseteq V_{i} \subseteq H$, we see that $H$ is normal indeed. As $U_{i}^{2} \subseteq V_{i}$, it follows that $U_{i} / V_{i}$ has exponent 2. For the orders we have $\left|U_{i}\right|=2^{7}$ and $\left|V_{i}\right|=2^{3}$, hence $\left|U_{i} / V_{i}\right|=2^{4}$.

Though we do not need it for the proof of our main result, we describe the lattice of normal subgroups of $G$. We shall use the following simple

LeMma 3.2. If $P$ is a finite p-group and $M$ is a maximal subgroup of $P$, then $[M, P]=P^{\prime}$.

Proof. Notice that $|P: M|=p$ and $M$ is normal in $P$. Now $M /[M, P] \subseteq \mathbf{Z}(P /[M, P])$ and $(P /[M, P]) /(M /[M, P])$ is cyclic (of order $p$ ), hence $P /[M, P]$ is abelian. Therefore we have $[M, P] \supseteq P^{\prime}$. The converse inclusion is obvious.

Proposition 3.3. The lattice of normal subgroups of $G$ is the union of the following nine intervals:

$$
I\left[G^{\prime}, G\right], \quad I\left[V_{i}, U_{i}\right](i=1, \ldots, 7), \quad I[\{1\}, \mathbf{Z}(G)] .
$$

Proof. Let $N$ be an arbitrary normal subgroup of $G$. We consider the product $N Z$. If $N Z=Z$, then $N$ is contained in $Z$. If $N Z=U_{i}$ for some $i$, $1 \leq i \leq 7$, then we have $N \supseteq N^{2}[N, G]=(N Z)^{2}[N Z, G]=U_{i}^{2}\left[U_{i}, G\right]=V_{i}$, so $N$ belongs to the interval $I\left[V_{i}, U_{i}\right]$. Finally, if $|G: N Z| \leq 2$, then $N \supseteq[N, G]=[N Z, G]=G^{\prime}$.

\section{Embeddings of $\mathscr{N}\left(Z_{p^{k}}^{n}\right)$.}

The following lemma about embeddings of the subgroup lattice of $Z_{p^{k}}^{n}$ $(n \geq 3)$ will play a central role in our proof. Parts of its statement are wellknown, but the freeness of $X$ may not have been observed before. 
Lemma 4.1. Let $\varphi: \mathscr{N}\left(Z_{p^{k}}^{n}\right) \rightarrow \mathscr{N}(A)$ be a lattice embedding, where $p$ is a prime, $k \geq 1, A$ is an abelian group, and assume that $\varphi(\{0\})=\{0\}$ and $\varphi\left(Z_{p^{k}}^{n}\right)=A$. If $n \geq 3$, then $A$ is isomorphic to a direct power $X^{n}$ of a free $Z_{p^{k-}}$ module $X$. Moreover, having identified $A$ and $X^{n}$,

$$
\varphi\left(\left\langle\left(z_{1}, \ldots, z_{n}\right)\right\rangle\right)=\left\{\left(z_{1} x, \ldots, z_{n} x\right) \mid x \in X\right\}
$$

holds for all $\left(z_{1}, \ldots, z_{n}\right) \in \mathbf{Z}_{p^{k}}^{n}$.

Proof. For notational simplicity we deal with the case $n=3$, the proof for $n>3$ is similar. Let $E_{1}=\langle(1,0,0)\rangle, E_{2}=\langle(0,1,0)\rangle, E_{3}=\langle(0,0,1)\rangle$, and $E_{0}=\langle(1,1,1)\rangle$. These subgroups form a spanning 3 -frame in $\mathscr{N}\left(\mathrm{Z}_{p^{k}}^{n}\right)$, hence so do their images in $\mathscr{N}(A)$. So by Lemma 1 of [HH2] we may assume that $A=X^{3}$ for some abelian group $X$, and we have $\varphi\left(E_{1}\right)=\{(x, 0,0) \mid$ $x \in X\}, \varphi\left(E_{2}\right)=\{(0, x, 0) \mid x \in X\}, \varphi\left(E_{3}\right)=\{(0,0, x) \mid x \in X\}$, and $\varphi\left(E_{0}\right)=$ $\{(x, x, x) \mid x \in X\}$. We introduce the notation $E_{i}^{*}=\varphi\left(E_{i}\right), i=0,1,2,3$.

In $[\mathrm{HH} 2]$ lattice terms $f_{j}(j=1,2, \ldots)$ are constructed such that $f_{j}\left(E_{0}^{*}, E_{1}^{*}, E_{2}^{*}, E_{3}^{*}\right)=\{(0, j x, x) \mid x \in X\}$ (and also $f_{j}\left(E_{0}, E_{1}, E_{2}, E_{3}\right)=\{(0, j t, t) \mid$ $\left.\left.t \in \mathbf{Z}_{p^{k}}\right\}\right)$. Since in $\mathbf{Z}_{p^{k}}^{n}$ we have $f_{p^{k}}\left(E_{0}, E_{1}, E_{2}, E_{3}\right)=E_{3}$, using the homomorphism $\varphi$ we obtain $f_{p^{k}}\left(E_{0}^{*}, E_{1}^{*}, E_{2}^{*}, E_{3}^{*}\right)=E_{3}^{*}$, i.e. $p^{k} X=0$, so $X$ can be considered as a $Z_{p^{k}}$-module. Our goal is to prove that $X$ is in fact a free $\mathbf{Z}_{p^{k}}$-module.

We have in $\mathscr{N}\left(\mathrm{Z}_{p^{k}}^{n}\right)$ :

$$
\langle(p, 0,0)\rangle=\langle(1,0,0)\rangle \cap\left\langle\left(1, p^{k-1}, 0\right)\right\rangle=E_{1} \cap f_{p^{k-1}}\left(E_{0}, E_{3}, E_{2}, E_{1}\right)
$$

and

$$
\begin{aligned}
\langle(p, 0,0)\rangle & =\langle(1,0,0)\rangle \cap(\langle(p, 1,0)\rangle+\langle(0,1,0)\rangle) \\
& =E_{1} \cap\left(f_{p}\left(E_{0}, E_{3}, E_{1}, E_{2}\right)+E_{2}\right) .
\end{aligned}
$$

Using the lattice homomorphism $\varphi$ we obtain

$$
E_{1}^{*} \cap f_{p^{k-1}}\left(E_{0}^{*}, E_{3}^{*}, E_{2}^{*}, E_{1}^{*}\right)=E_{1}^{*} \cap\left(f_{p}\left(E_{0}^{*}, E_{3}^{*}, E_{1}^{*}, E_{2}^{*}\right)+E_{2}^{*}\right),
$$

that is

$$
\begin{gathered}
\{(x, 0,0) \mid x \in X\} \cap\left\{\left(y, p^{k-1} y, 0\right) \mid y \in X\right\} \\
=\{(u, 0,0) \mid u \in X\} \cap(\{(p v, v, 0) \mid v \in X\}+\{(0, w, 0) \mid w \in X\}),
\end{gathered}
$$

so

$$
\left\{(x, 0,0) \mid x \in X, p^{k-1} x=0\right\}=\{(p v, 0,0) \mid v \in X\},
$$


from which it follows that $X$ is a free $Z_{p^{k}}$-module. Thus we can assume that $X$ is equal to a direct sum of some, say $\kappa$, copies of $Z_{p^{k}}$ 's.

It remains to show (1). For every element

$$
\mathbf{u}=\left(\ldots, u_{i}, \ldots\right) \in \bigoplus_{\kappa} \mathbf{z}_{p^{k}}^{3}
$$

with

$$
u_{i}=\left(u_{i}^{1}, u_{i}^{2}, u_{i}^{3}\right) \in \mathrm{Z}_{p^{k}}^{3}
$$

define

$$
\rho(\mathbf{u})=\left(\left(\ldots, u_{i}^{1}, \ldots\right),\left(\ldots, u_{i}^{2}, \ldots\right),\left(\ldots, u_{i}^{3}, \ldots\right)\right) \in X^{3} .
$$

Clearly, $\rho: \bigoplus_{\kappa} Z_{p^{k}}^{3} \rightarrow X^{3}$ is a group-isomorphism. For a subgroup $U \subseteq Z_{p^{k}}^{3}$ let $\psi(U) \subseteq X^{3}$ be the image of $\bigoplus_{\kappa} U$ under $\rho$. Then $\psi$ is a lattice embedding of $\mathscr{N}\left(Z_{p^{k}}^{3}\right)$ into $\mathscr{N}\left(X^{3}\right)$. An easy calculation shows that (1) holds, when $\varphi$ is replaced by $\psi$. In particular, $\psi\left(E_{i}\right)=\varphi\left(E_{i}\right)$ for $i=0,1,2,3$. It is shown in [HH1] (see Section 3.2) that the 3-frame $E_{0}, E_{1}, E_{2}, E_{3}$ generates $\mathscr{N}\left(\mathrm{Z}_{p^{k}}^{3}\right)$. Therefore $\psi=\varphi$, proving (1).

COROLlaRY 4.2. For every subgroup $U$ of $\mathrm{Z}_{p^{k}}^{n}$ and for every positive integer $m$ we have $\varphi(m U)=m \varphi(U)$.

Proof. By (1) the claim is obvious for cyclic subgroups $U$. If $U$ is arbitrary, then write it as a sum of cyclic subgroups $U=\sum C_{i}$. Then it follows easily that $\varphi(m U)=\varphi\left(m \sum C_{i}\right)=\varphi\left(\sum m C_{i}\right)=\sum \varphi\left(m C_{i}\right)=\sum m \varphi\left(C_{i}\right)=$ $m \sum \varphi\left(C_{i}\right)=m \varphi\left(\sum C_{i}\right)=m \varphi(U)$.

\section{Proof of the Theorem.}

Let us assume, by way of contradiction, that there exists an embedding of $\mathscr{N}(G)$ into $\mathscr{N}(A)$ for some abelian group $A$. Let us denote the image of a normal subgroup $N$ of $G$ under this embedding by $N^{*}$. Without loss of generality we may suppose that $\{1\}^{*}=\{0\}$ and $G^{*}=A$.

The interval between $\{1\}$ and $Z=\mathbf{Z}(G)$ in $\mathscr{N}(G)$ is isomorphic to $\mathscr{N}\left(Z_{2}^{6}\right)$ and the interval between $Z$ and $G$ is isomorphic to $\mathscr{N}\left(Z_{2}^{3}\right)$. Hence by Lemma 4.1 both $Z^{*}$ and $A / Z^{*}$ have exponent 2 , hence the exponent of $A$ divides 4 , so in other words $A$ is a $Z_{4}$-module. Moreover, the interval between $D=G^{\prime}$ and $G$ is isomorphic to $\mathscr{N}\left(Z_{4}^{3}\right)$, hence - again by Lemma 4.1 it follows that $A / D^{*}$ is a free $Z_{4}$-module. Since free modules are projective, we have that $A=D^{*} \oplus B$ for some subgroup $B$. Note that $2 D^{*}=0$, hence $2 A=2 B$. 
Now take the normal subgroups $U_{i}, V_{i}$ defined in Section 3. Since $U_{i} / V_{i}$ is elementary abelian of order $2^{4}$, another application of Lemma 4.1 yields that $U_{i}^{*} / V_{i}^{*}$ has exponent 2, i.e. $2 U_{i}^{*} \subseteq V_{i}^{*}$. We also have $2 U_{i}^{*} \subseteq 2 A=2 B$.

Consider the embedding $\psi$ of $\mathscr{N}(G / D) \cong \mathscr{N}\left(Z_{4}^{3}\right)$ into $\mathscr{N}\left(A / D^{*}\right)$ induced by *. Applying Corollary 4.2 we get that $2 \psi\left(U_{i} D / D\right)=\psi\left(2\left(U_{i} D / D\right)\right)$. But we have $V_{i} D=U_{i}^{2} D$ in $G$, so $2\left(U_{i} D / D\right)=V_{i} D / D$. Thus, $\left(D^{*}+V_{i}^{*}\right) / D^{*}=$ $\psi\left(V_{i} D / D\right)=2\left(\left(D^{*}+U_{i}^{*}\right) / D^{*}\right)$, hence

$$
D^{*}+V_{i}^{*}=D^{*}+2 U_{i}^{*} .
$$

From $A=D^{*} \oplus B$ we get for every $C \supseteq D^{*}$ that $C=D^{*} \oplus(B \cap C)$. The mapping $C \mapsto(B \cap C)$ is a lattice isomorphism $\mathscr{N}\left(A / D^{*}\right) \rightarrow \mathscr{N}(B)$. Denote by $\psi^{\prime}: \mathscr{N}(G / D) \rightarrow \mathscr{N}(B)$ the embedding obtained from $\psi$ by composing it with this isomorphism. Applying Corollary 4.2 again we get that $\psi^{\prime}(2(G / D))=2 \psi^{\prime}(G / D)$. We have seen that $2(G / D)=Z / D$, so from $\psi^{\prime}(G / D)=B$ we obtain that $\psi^{\prime}(Z / D)=2 B$. On the other hand, $\psi^{\prime}(Z / D)=Z^{*} \cap B$. Therefore we get that

$$
Z^{*}=D^{*} \oplus 2 B \text {. }
$$

Notice that we do not claim that $2 B$ or $2 U_{i}^{*}$ corresponds to any normal subgroup of $G$.

We will show that

$$
V_{i}^{*}=\left(D^{*} \cap V_{i}^{*}\right) \oplus\left(2 B \cap V_{i}^{*}\right)
$$

holds for each $1 \leq i \leq 7$. Let us denote the right-hand side of the equation by $W_{i}$. We obviously have $V_{i}^{*} \supseteq W_{i}$. We take intersection and sum of both $V_{i}^{*}$ and $W_{i}$ with $D^{*}$. The equation

$$
D^{*} \cap W_{i}=D^{*} \cap V_{i}^{*}
$$

is trivial. On the other hand, we have

$$
D^{*}+W_{i}=D^{*} \oplus\left(2 B \cap V_{i}^{*}\right) \supseteq D^{*} \oplus 2 U_{i}^{*}=D^{*}+V_{i}^{*} .
$$

By modularity, we infer that $V_{i}^{*}=W_{i}$, indeed.

We will reach the contradiction by showing that no such subgroup $K$ exists for which $Z^{*}=D^{*} \oplus K$ and $V_{i}^{*}=\left(D^{*} \cap V_{i}^{*}\right) \oplus\left(K \cap V_{i}^{*}\right)$ for all $1 \leq i \leq 7$ hold. Using the basis $[a, b],[a, c],[b, c], a^{2}, b^{2}, c^{2}$ of $Z$, Lemma 4.1 provides a decomposition $Z^{*} \cong X^{6}$ such that we have 


$$
\begin{aligned}
D^{*} & =\{(x, y, z, 0,0,0) \mid x, y, z \in X\}, \\
V_{1}^{*} & =\{(x, y, 0, t, 0,0) \mid x, y, t \in X\}, \\
V_{2}^{*} & =\{(x, 0, y, 0, t, 0) \mid x, y, t \in X\}, \\
V_{3}^{*} & =\{(0, x, y, 0,0, t) \mid x, y, t \in X\}, \\
V_{4}^{*} & =\{(x, y, y, t, t, 0) \mid x, y, t \in X\}, \\
V_{5}^{*} & =\{(x, y, x, t, 0, t) \mid x, y, t \in X\}, \\
V_{6}^{*} & =\{(x, x, y, 0, t, t) \mid x, y, t \in X\}, \\
V_{7}^{*} & =\{(x+y+t, x+t, y+t, t, t, t) \mid x, y, t \in X\} .
\end{aligned}
$$

Now $\quad D^{*} \cap V_{1}^{*}=\{(x, y, 0,0,0,0) \mid x, y \in X\}, \quad$ hence $\quad V_{1}^{*}=\left(D^{*} \cap V_{1}^{*}\right) \oplus$ $\left(K \cap V_{1}^{*}\right)$ implies that

$$
V_{1}^{*} \cap K=\left\{\left(\alpha_{11} t, \alpha_{12} t, 0, t, 0,0\right) \mid t \in X\right\}
$$

for suitable maps $\alpha_{11}, \alpha_{12}$ from $X$ to $X$. It is easy to check that in fact $\alpha_{11}$ and $\alpha_{12}$ are endomorphisms of $X$. Similarly,

$$
\begin{aligned}
& V_{2}^{*} \cap K=\left\{\left(\alpha_{21} t, 0, \alpha_{23} t, 0, t, 0\right) \mid t \in X\right\}, \\
& V_{3}^{*} \cap K=\left\{\left(0, \alpha_{32} t, \alpha_{33} t, 0,0, t\right) \mid t \in X\right\} .
\end{aligned}
$$

Hence we have

$$
K \supseteq\left\{\left(\alpha_{11} r+\alpha_{21} s, \alpha_{12} r+\alpha_{32} t, \alpha_{23} s+\alpha_{33} t, r, s, t\right) \mid r, s, t \in X\right\} .
$$

From $Z^{*}=D^{*} \oplus K$ it follows that we have equality here. Then

$$
V_{4}^{*} \cap K=\left\{\left(\left(\alpha_{11}+\alpha_{21}\right) r, \alpha_{12} r, \alpha_{23} r, r, r, 0\right) \mid r \in X, \alpha_{12} r=\alpha_{23} r\right\} .
$$

From the direct decomposition $V_{4}^{*}=\left(D^{*} \cap V_{4}^{*}\right) \oplus\left(K \cap V_{4}^{*}\right)$ we infer that $\alpha_{12} r=\alpha_{23} r$ holds for every $r \in X$, i.e. $\alpha_{12}=\alpha_{23}$. Similarly, calculating $V_{5}^{*} \cap K$ and $V_{6}^{*} \cap K$ we obtain $\alpha_{11}=\alpha_{33}$ and $\alpha_{21}=\alpha_{32}$. Finally, using that $2 X=0$ we get

$$
\begin{array}{r}
V_{7}^{*} \cap K=\left\{\left(\left(\alpha_{11}+\alpha_{21}\right) t,\left(\alpha_{12}+\alpha_{21}\right) t,\left(\alpha_{12}+\alpha_{11}\right) t, t, t, t\right) \mid t \in X,\right. \\
\left.\left(\alpha_{11}+\alpha_{21}\right) t+\left(\alpha_{12}+\alpha_{21}\right) t+\left(\alpha_{12}+\alpha_{11}\right) t+t=0\right\} .
\end{array}
$$

The latter condition means $t=0$, so $V_{7}^{*} \cap K=\{0\}$, a contradiction. This proves our theorem.

\section{REFERENCES}

[A] M. Aschbacher, Finite Group Theory, Cambridge Univ. Press, Cambridge, 1986.

[B] G. Birkhoff, Lattice Theory, Amer. Math. Soc. Providence, RI, 1967. 
[HH1] C. Herrmann and A. P. Huhn, Lattices of normal subgroups which are generated by frames, Colloq. Math. Soc. J. Bolyai 14 (1974), 97-136.

[HH2] C. Herrmann und A. P. Huhn, Zum Begriff der Charakteristik modularer Verbände, Math. Z. 144 (1975) 185-194.

[J] B. Jónsson, On the representation of lattices, Math. Scand. 1 (1953), 193-206.

[M] R. McKenzie, Some interactions between group theory and the general theory of algebras, Groups - Canberra 1989 (ed. L. G. Kovács), Springer Lecture Notes in Math. 1456 (1990), 32-48.

[PSz1] P. P. Pálfy and Cs. Szabó, An identity for subgroup lattices of abelian groups, Algebra Universalis 33 (1995), 191-195.

[PSz2] P. P. Pálfy and Cs. Szabó, Congruence varieties of groups and abelian groups, in: Lattice Theory and Its Applications (eds. K.A. Baker and R. Wille), Heldermann Verlag (1995), 163-183.

EÖTVÖS UNIVERSITY

DEPARTMENT OF ALGEBRA AND NUMBER THEORY

BUDAPEST

HUNGARY

Email: ewkiss@cs.elte.hu

MATHEMATICAL INSTITUTE OF THE HUNGARIAN ACADEMY OF SCIENCES

BUDAPEST

HUNGARY

Email:ppp@math-inst.hu 